\title{
Esophageal subepithelial tumor: why tunneling?
}

\begin{abstract}
Subepithepial tumors (SETs) in esophagus are mostly benign and causes no specific symptoms, however, resection should be considered in case of large tumors leading to troublesome symptoms such as dysphagia or rapidly growing tumor. Endoscopioc submucosal dissection (ESD) has been performed for therapeutic resection of esophageal epithepial lesions or even SETs, however, post-procedureal stricture is a major concern. Recently, a novel endoscopic approach using submucosal space, namely submucosal tunneling, is considered as a good alternative option for endoscopic resection of esophageal lesions. This approach can preserve mucosal integrity and use it as a coverage flap, which prevents serious late complications such as stricture in esophagus. Here, we report two cases of large esophageal SETs which were endoscopically resected by two different approaches: the first one by conventional ESD, and the second one by submucosal tunneling approach. The former eventually resulted in significant stricture at esophagus, thus repetitive balloon dilation was needed. However, the latter had no serious late complications including stricture. This case report clearly provides the advantage of submucosal tunneling over conventional ESD in the treatment of esophageal SETs, and the promising therapeutic value of this new approach is expected.
\end{abstract}

Keywords: subepithelial tumor, submucosal tunneling, endoscopic submucosal dissection
Volume 5 Issue 4 - 2016

\author{
Sung Ho Kim,' Moon Kyung Joo,' Jong Jae \\ Park,' Beom Jae Lee,' Hoon Jai Chun,' Sang \\ Woo Lee,' Sun Suk Kim, ${ }^{2}$ Young Tae Bak' \\ 'Department of Internal Medicine, Korea University College of \\ Medicine, Republic of Korea \\ ${ }^{2}$ Health Promotion Center, Korea University, Republic of Korea
}

Correspondence: Jong-Jae Park, MD, Ph.D, Division of Gastroenterology, Department of Internal Medicine, Korea University College of Medicine Guro Hospital. I48, Gurodongro, Guro-gu, Seoul, 152-703, Republic of Korea, Tel 82-2-26261027, Fax 82-2-2626-2024, Email gi7pjj@korea.ac.kr

Received:September 01, 2016 | Published: October 06, 2016

\section{Introduction}

Endoscopic submucosal dissection (ESD) has been widely accepted as a standard therapeutic tool for epithelial neoplasm in digestive tract, and esophagus is no exception. However, as technical skills advanced and clinical experiences accumulated, endoscopists set up new approaches beyond intraluminal and extraluminal space, namely, "the third space". This "third space" remains virtual and has to be created by dissecting and expanding the tissue layer between the mucosa and the muscularis propria, allowing the endoscope to gain access. Submucosal tunneling by the ESD technique has been demonstrated as technically feasible and effective as an access method, and especially, the submucosal tunnel, which is a space that lay within the gut wall, is an inspired and novel arena or space for endoscopic maneuvers. ${ }^{1}$ Here, we report two cases of large esophageal subepithelial tumors (SETs); the one was resected by conventional ESD techinique, and the other by submusal tunneling method, and discuss the potential of submucosal endoscopy as a promosing therapeutic tool.

\section{Case presentation}

\section{Case I}

A 42-year -old woman was refferred to our hospital for further evaluation of dysphagia. The patient had no specific past history, and physical examination showed no remarkable findings. Laboratory examination also showed all negative findings. An esophagogastroduodenoscopy (EGD) was performed, and revealed a large, $4 \mathrm{~cm}$ sized, elongated SET at upper to mid esophagus, 23 27 $\mathrm{cm}$ from incisor (Figure 1A). An endoscopic ultrasonography (EUS) was performed to further evaluate the nature and origin of esophageal SET, which showed a homogenous hypoeshoic mass originated from muscularis propria layer (Figure 1B). We decided to perform endoscopic resection of esophaegeal SET for relief of symptom, and conventional ESD technique was used. ${ }^{2}$ Briefly, lesion boundaries were identified and marked using argon plasma coagulation connected to an electrosurgical generator (Erbe ICC 200W; Erbe Elektromedizin $\mathrm{GmbH}$, Tübingen, Germany). After injection of a $0.9 \%$ saline solution mixed with epinephrine (dilution rate 1:10,000) and indigo carmine dye into the submucosal layer, an initial incision was made $3 \sim 5 \mathrm{~mm}$ from the regular mucosal layer in a regular interval using needle knife (KD-IL-1, Olympus Optical Co. Ltd.). Submucosal dissection was done with insulated-tipped electrosurgical knife (IT knife) (KD-610L, Olympus Optical Co Ltd.). Dissection of the tumor was performed successfully, however, a large mucosal defect was noted after the procedure (Figure 2A-2D). The resected specimen was $4.2 \mathrm{~cm}$ in length and $1.5 \mathrm{~cm}$ in diameter, and tumor was diagnosed as leiomyoma by histopathologic examination. The patients had no immediate major complications, and discharged 3 days after the procedure. However, 2 weeks later, the patient complained of significant chest discomfort and dysphagia, and a follow-up EGD showed significant stricture at the procedure site where the scope could not be passed. Endoscopic balloon dilatation was performed and repeated 8 times over 4 months, which led to the resolution of the stricture (Figure 3A-3C).

\section{Case 2}

A 61-year -old woman was refferred to our hospital for further evaluation of esophageal SET. She hassuffered fromdysphagiafor many years, and an EGD showed a large, $3.5 \mathrm{~cm}$ sized, elongated SET at upper esophagus, $22 \mathrm{~cm}$ from incisor, and it showed positive rolling sign (Figure 4). As the conventional ESD is expected to cause postprocedural stricture of esophagus, we decided to perform subepithelial tunnelling endoscopic resection (STER) method to preserve mucosal integrity and prevent stricture. STER was performed according to previously suggested protocols. ${ }^{3}$ Briefly, a mixed solution of epinephrine in saline $(1: 10,000)$ was injected at submucosal layer, and a $2.0 \mathrm{~cm}$ longitudinal mucosal incision was made to gain entry to the submucosal area and a submucosal tunnel was created $5.0 \mathrm{~cm}$ proximal to the SET until the tumor was visible by an endoscope while carefully preserving the covering mucosal integrity After exposure of the SET, complete dissection of the tumor was carefully 
performed using an IT knife along the margin. After dissection, mucosal integrity was preserved well, and the mucosal entry site was closed with standard hemostatic clips (HX-610-90, Olympus Optical Co Ltd.) (Figure 5A-5D). The resected specimen was $3.5 \mathrm{~cm}$ in length and $1.7 \mathrm{~cm}$ in diameter, and tumor was diagnosed as leiomyoma by histopathologic examination. The patietns had no immediate major complications, and discharged 3 days after the procedure. A follow-up EGD was performed at 6 and 24 months after the procedure, which showed no significant stricture, and only scar change was noted on the mid esophagus (Figure 6).


Figure I Esophageal subepithelial tumor (SET).

(A) An esophagogstroduodenoscopy (EGD) showed a large $(4.0 \mathrm{~cm})$ SET at upper to mid esophagus. (B) An endoscopic ultrasonography showed a large hypoeshoic mass originated from muscularis propria layer.



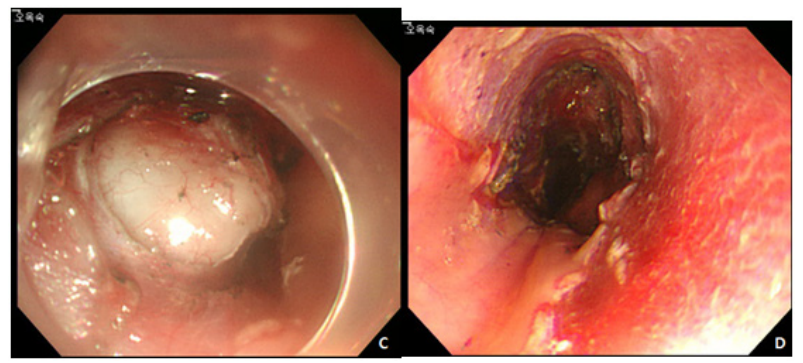

Figure 2 Endoscopic submucosal dissection (ESD) of esophageal subepithelial tumor (SET).

(A) At initial,an incision was made from the regular mucosal layer. (B, C) Esopahgeal SET was successfully resected by using conventional ESD method. (D) A large muocsal defect was noted after the procedure.
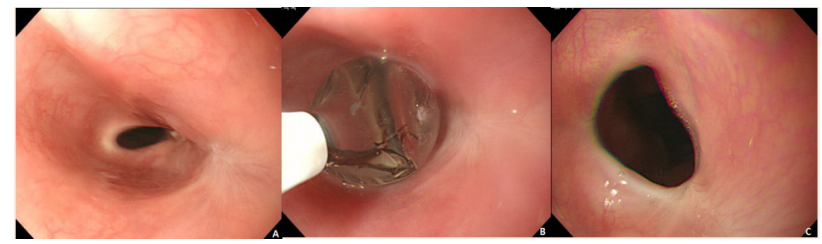

Figure 3 Endoscopic treatment of esophageal stricture.

(A) A follow-up esophagogastroduodenoscopy (EGD) at I month after the procedure revealed significant stricture at esophagus. (B) Repeatitive endoscopic balloon dilation was performed. (C) After 8 times of endoscopic ballooning, esophageal stricture was resolved.

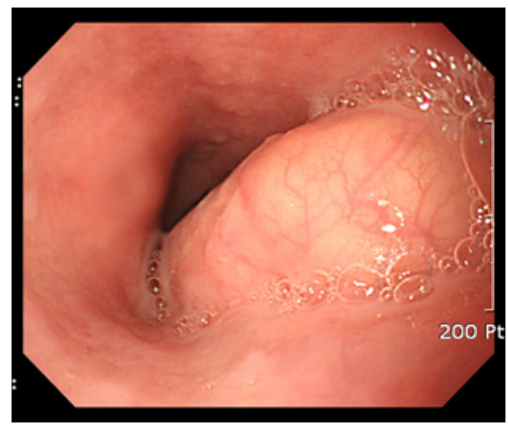

Figure $4 \mathrm{~A}$ large esophageal subepithelial tumor (SET).

An esophagogstroduodenoscopy (EGD) showed a large $(3.5 \mathrm{~cm})$ SET at mid esophagus.

\section{Discussion}

Subepithelial lesions in esophagus are mostly benign, and leiomyoma is the most common accounting about two-thirds of all benign tumors of esophagus, followed by grannular cell tumor and hemangioma.$^{4}$, and malignant lesion among esophageal SETs are exterely rare. ${ }^{5}$ Furthermore, majority of esophageal SETs are incidental findings and do not cause any specific symptoms, thus do not need to be resected. However, if a specific symptom such as dysphagia develops by a large esophageal SET or a tumor shows rapidly growing pattern, endoscopist should consider resection of tumor for relief of patient's symptom and pathologoic cofirmation. ESD has been performed as therapeutic procedure for the treatment of esophageal superficial neoplasms or even SETs, and is considered to have several advantages over surgical procedure in terms of safety especially when patients have severe comorbidities. ${ }^{6}$ However, post-ESD stricture is one of major complications which can lead to deterioration of patient's symptom and additional procedure to resolve it. ${ }^{7}$ Furthermore, preventive strategy to reduce significant 
stricture after ESD of esophageal SETs is still lacking. In this situation, submucoal tunneling method including STER may have superiority over conventional ESD. In concrete, first, the integrity of the digestive tract mucosa and submucosa can be preserved to a degree by closure of the flap entry site with multiple clips, which can enhance wound healing. Second, a 5-cm-long submucosal tunnel has a good leak-proofing effect, and if digestive tract leakage occurs during the procedure, it reduces the risk of fistula formation or pleural \& abdominal infection. Third, STER enables identification of the tumor layer of origin. And lastly, bleeding can be immediately detected and successfully controlled with precise hemostasis, which also reduces the risk of massive or delayed bleeding. ${ }^{8}$ However, technical difficulties of maintaining mucoal integrity within a narrow working space is a major concern of successful STER procedure. Several previous case series studies reported the successful removal of esophageal SETs by using STER procedure, which showed maximal diameter of tumors ranged 19-29 mm and technical were success rate $83-100 \%$. Major complicationssuch as pneumomediastinum were found in $0-17 \%$, which could be managed by conservative management. ${ }^{9-11}$ Our cases clearly show the different post-procedural outcomes between conventional ESD and STER in esophageal SETs, and we consider that tunneling method may replace the conventional ESD for the therapeutic endoscopic procedure of large esophageal SETs in the near future.

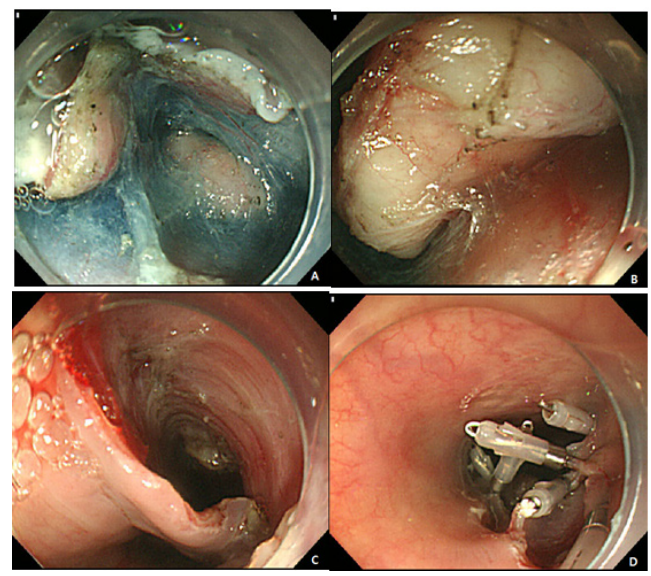

Figure 5 Submucosal tunneling endoscopic resection (STER) of esophageal subepithelial tumor (SET).

(A) Submucosal tunneling was made to approach and expose the tumor. (B) Dissection of the tumor was performed by using an insulated-tip knife. (C) After resection of the tumor, mucosal integrity was preserved as flat. (D) Entry site was sealed by using endoscopic clips.

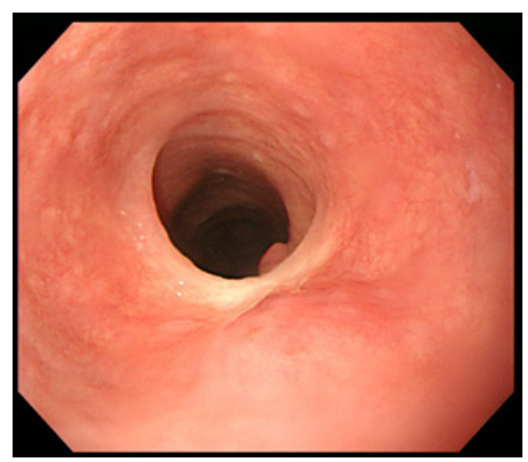

Figure 6 A follow-up esophagogastroduodenoscopy (EGD) after submucosal tunneling endoscopic resection of esophageal subepithelial tumor. No significant stricture was developed by a follow-up EGD at 24 months after the procedure.

\section{Acknowledgments}

None.

\section{Conflicts of interest}

The authors declare no conflicts of interest.

\section{Funding}

None.

\section{References}

1. Kobara H, Mori H, Rafiq $\mathrm{K}$, et al. Submucosal tunneling techniques:Current perspectives. Clin Exp Gastroenterol. 2014;7:67-74.

2. Min BH, Kim ER, Kim KM, et al. Surveillance strategy based on the incidence and patterns of recurrence after curative endoscopic submucosal dissection for early gastric cancer. Endoscopy. 2015;47(9):784-793.

3. Joo MK, Park JJ, Kim H, et al. Endoscopic versus surgical resection of gi stromal tumors in the upper gi tract. Gastrointest Endosc 2016;83(2):318-326.

4. Tsai SJ, Lin CC, Chang CW, et al. Benign esophageal lesions: Endoscopic and pathologic features. World J Gastroenterol. 2015;21(4):1091-1098.

5. Kim SB, Lee SH, Gu MJ. Esophageal subepithelial lesion diagnosed as malignant gastrointestinal neuroectodermal tumor. World $J$ Gastroenterol. 2015;21(18):5739-5743.

6. Park JS, Youn YH, Park JJ, et al. Clinical outcomes of endoscopic submucosal dissection for superficial esophageal squamous neoplasms. Clin Endosc. 2016;49(2):168-175.

7. Jain D, Singhal S. Esophageal stricture prevention after endoscopic submucosal dissection. Clin Endosc. 2016;49(3):241-256.

8. Liu BR, Song JT. Submucosal tunneling endoscopic resection (ster) and other novel applications of submucosal tunneling in humans. Gastrointest Endosc Clin N Am. 2016;26(2):271-282.

9. Inoue H, Ikeda H, Hosoya T, et al. (2012) Submucosal endoscopic tumor resection for subepithelial tumors in the esophagus and cardia. Endoscopy. 2012;44(3):225-230.

10. Xu MD, Cai MY, Zhou PH, et al. Submucosal tunneling endoscopic resection:A new technique for treating upper gi submucosal tumors originating from the muscularis propria layer (with videos). Gastrointest endosc. 2012;75(1):195-199.

11. Gong W, Xiong Y, Zhi F, et al. Preliminary experience of endoscopic submucosal tunnel dissection for upper gastrointestinal submucosal tumors. Endoscopy. 2012;44(3):231-235. 